\title{
Lin28/microRNA-let-7a promotes metastasis under circumstances of hyperactive Wnt signaling in esophageal squamous cell carcinoma
}

\author{
RUI LING ${ }^{1 *}$, YUEPENG ZHOU ${ }^{1 *}$, LING ZHOU ${ }^{1}$, DONGFANG DAI ${ }^{1}$, DAN WU ${ }^{1}$, \\ LEI MI ${ }^{1}$, CHAOMING MAO ${ }^{1,2}$ and DEYU CHEN ${ }^{1}$ \\ ${ }^{1}$ Institute of Oncology; ${ }^{2}$ Department of Nuclear Medicine, \\ Affiliated Hospital of Jiangsu University, Zhenjiang, Jiangsu 212001, P.R. China
}

Received September 4, 2017; Accepted January 19, 2018

DOI: $10.3892 / \mathrm{mmr} .2018 .8548$

\begin{abstract}
Dysregulation of micro (mi)RNA-let-7 has been associated with the development and prognosis of multiple cancer types. Lin28, a RNA-binding protein, plays a conserved role in regulating the maturation of let-7 family proteins. However, few studies have focused on the effects of Lin28/let-7 on Wnt-activated esophageal squamous cell carcinoma (ESCC). Analysis of the expression of let-7a, let-7b and let-7c in clinical tissues revealed that lower let-7a expression was correlated with higher tumor node metastasis staging and recurrence in patients with ESCC. Furthermore, it was demonstrated that let-7a was inversely correlated with the migration and invasion of ESCC cells. In addition, epithelialmesenchymal transition, and the expression of VEGF-C and MMP9 were effectively decreased by let-7a-mimic or siRNALin 28 pretreatment. Mechanistically, Lin28 functioned as the key factor in signal transduction, which regulated the expression of let-7a and the downstream genes along the Wnt signaling pathway. Taken together, these findings identified a biochemical and functional association between Lin28/let-7a, and the Wnt pathway in ESCC cells.
\end{abstract}

Correspondence to: Professor Deyu Chen, Institute of Oncology, Affiliated Hospital of Jiangsu University, 438 Jie-Fang Road, Zhenjiang, Jiangsu 212001, P.R. China

E-mail: cdeyu@hotmail.com

Professor Chaoming Mao, Department of Nuclear Medicine, Affiliated Hospital of Jiangsu University, 438 Jie-Fang Road, Zhenjiang, Jiangsu 212001, P.R. China

E-mail: jq1001@ujs.edu.cn

*Contributed equally

Key words: miRNA-let-7a, Lin28, metastasis, Wnt, esophageal squamous carcinoma

\section{Introduction}

Esophageal cancer (EC) is the most common gastrointestinal tumor worldwide, with the highest incidence and mortality rates observed in China, where most cases are esophageal squamous cell carcinoma (ESCC) (1), and as the fourth cause of cancer death, the 5-year survival rate remains at $15-40 \%$ after current treatments (2-4). Indeed, the main causes of poor prognosis for ESCC patients are local invasion and lymph node and distant metastasis; thus, early detection is a key factor in increasing survivability (3), although the lack of effective and specificity markers still plagues the diagnosis. Of note, since carcinogenesis is a multi-step process, the diagnostic indices, such as epithelial mesenchymal transition (EMT), invasion, metastases, and recurrence, should dynamically change with the occurrence and development of tumors (5).

For squamous neoplasms, such as ESCCs, EMT is a crucial process, in which cancer cells lose polarity and reduce adhesion, contributing to migration to and invasion of surrounding issues (6). Therefore, discovering the mechanism and key factors that control EMT in tumors would provide strategies to solve the above dilemma. Recent data have demonstrated that microRNAs (miRNAs) as non-coding small RNAs not only exist widely in organisms, but also govern the expression of different genes by binding to the 3'-untranslated region of target genes and participating in a series of important processes, including differentiation, proliferation, apoptosis and EMT (7-9). However, study results are conflicting, and microRNAs play roles as tumor-suppressors or carcinogenic factors in various human malignancies (10). Studies have shown that miRNA-let-7 as a tumor suppressor, apparently has low expression in many tumor types, such as head and neck, gastric, lung, ovarian and esophageal cancers (11-16). Among the let-7 family, let-7a, have been implicated in the inhibition of EMT in nasopharyngeal, hepatocellular and rectal carcinoma, and breast cancer (17-20). Specifically, as a highly conserved RNA-binding protein, Lin28, which negatively regulates the maturation of let-7 by binding to the terminal loop of its precursor, has also been positively correlated with the cancer aggressiveness and poor prognosis of EC patients $(12,16,17)$. Therefore, it is worth considering whether the changes of let- $7 \mathrm{a}$ 
have a potential indicative effect of EMT, even metastasis for ESCC.

At this stage, the occurrence of EMT involves multiple signal transduction pathways, such as Notch, TNF- $\alpha$, TGF- $\beta$, Wnt/ $\beta$-catenin and others in tumors $(19,21)$. Given the many research theories in existence, there can be no doubt that the Wnt signaling plays a critical role in deciding the destiny of cancer cells. It has now been definitively established that $\beta$-catenin migrates to the nucleus, interacts with transcription factors $\mathrm{TCF} / \mathrm{LEF}$, and regulates the expression of downstream target genes, such as cyclin D1, c-myc, etc., thereby inducing a series of biological functions including self-renewal, differentiation, proliferation and migration (22). However, controversial evidence suggests that Wnt signaling consists of a series of oncogene and tumor-suppressor genes. Nonetheless, the cascading effect of downstream target miRNAs has attracted much attention in recent years (23). Regarding ESCC, our previous study identified that interleukin-23 (IL-23) promotes EMT via the Wnt/ $\beta$-catenin pathway (24). Additionally, recent research revealed that the Wnt pathway could cooperate with Lin28, and the abnormal activation of $\mathrm{Wnt} / \beta$-catenin signaling plays a significant role in the metastasis and recurrence of esophageal cancer $(16,19,25-27)$. However, there is currently no evidence that let-7a acts as a prognostic indicator via Wnt/Lin28 regulation in ESCC.

In this study, we aimed to explore the role of Lin28/let-7a in regulation of migration, invasion and metastasis in ESCC. We found that let-7a, out with other members, was negatively correlated with higher TNM staging and recurrence of ESCC patients. Moreover, we showed that Lin28 knock-down or let-7a mimic repressed the invasion, EMT and metastasis. Furthermore, we identified an important role of Wnt signaling in the maintaining Lin 28 of ESCC cells. We thus identify an important biochemical and functional link between Lin28/let-7a with the Wnt pathway in ESCC progression.

\section{Materials and methods}

Patients and tsissues. The Medical Ethics Committee of Jiangsu University has approved this study. A total of 70 tumors and para-carcinoma tissues were obtained from patients who had ESCC, had not undergone chemotherapy or radiotherapy, had signed informed consent before surgical resection, and had data that were collected from 2011 to 2013 at the Affiliated Hospital of Jiangsu University (Zhenjiang, China). All tissue samples were confirmed by independent pathologists, frozen in liquid nitrogen, and stored at $-80^{\circ} \mathrm{C}$. Patients who developed any local recurrence or distant metastasis diagnosed by computerized tomography scan within 3 years after therapy are defined as recurrence (28).

Cell lines and RNA interference. The ESCC cell lines TE-1, ECA109 and KYSE-150, and normal esophageal squamous epithelium cell line Het-1a were purchased from the Cell Bank of the Chinese Academy of Sciences (Shanghai, China). All cells were cultured in RPMI-1640 medium containing 10\% FBS, $100 \mathrm{IU} / \mathrm{ml}$ penicillin and streptomycin at $37^{\circ} \mathrm{C}, 5 \%$ $\mathrm{CO}_{2}$. All media were purchased from Gibco (Grand Island, NY, USA). The following siRNA sequences were designed and synthesized by GenePharma Company (GenePharma,
Shanghai, China): let-7a mimics 5'-UGAGGUAGUAGGUUG UAUAGUU-3' (forward), 5'-CUAUACAACCUACUACCU CAUU-3' (reverse); negative control (NC) for mimics 5'-UUC UCCGAACGUGUCACGUTT-3' (forward), 5'-ACGUGA CACGUUCGGAGAATT-3' (reverse); let-7a inhibitor 5'-AAC UAUACAACCUACUACCUCA-3'; NC for inhibitor 5'-CAG UACUUUUGUGUAGUACAA-3'. Mimics (50 nM) or inhibitors $(100 \mathrm{nM})$ were transfected into ECA-109 cell lines with Lipofectamine 2000 reagents (Invitrogen, Shanghai, China) according to the manufacturer's instructions. Untransfected groups were cultured under normal conditions.

Molecular biology experiments. Total RNA was extracted from ESCC tissues and cells with TRIzol reagent (Invitrogen, Carlsbad, CA, USA) according to the manufacturer's instructions. Relative concentrations of RNA were quantified using the BioMate 3S Analyzer (Thermo Fisher Scientific, Inc., San Jose, CA, USA), normalized to the expression of U6 or GAPDH, and performed using a GeneAmp ${ }^{\circledR}$ PCR systems 9700 (Bio-Rad, Hercules, CA, USA). The following sequences of PCR primers (Invitrogen, Shanghai, China) were used: let-7a (forward, 5'-GCCGCTGAGGTAGTAGGTTGTA-3' and reverse, 5'-GTG CAGGGTCCGAGGT-3'); let-7b (forward, 5'-TGAGGTAGT AGGTTGTGTGGTT-3' and reverse, 5'-GCTGTCAACGAT ACGCTACCTA-3'); let-7c (forward, 5'-ACACTCCAGCTG GGTGAGGTAGTAGGTT-3' and reverse, 5'-GGTGTCGTG GAGTCG-3'); U6 (primers: 5'-AACGCTTCACGAATT TGCGT-3', forward, 5'-CTCGCTTCGGCGCAGCACA-3' and reverse, 5'-AACGCTTCACGAATTTGCGT-3'); Lin28 (forward, 5'-AAAGGAGACAGGTGCTAC-3' and reverse, 5'-ATATGGCTGATGCTCTGG-3'); Snail (forward, 5'-CTT CTCCTCTACTTCAGTCTCTTCC-3' and reverse, 5'-TGAGGT ATTCCTTGTTGCAGTATTT-3'); Slug (forward, 5'-AACAGA GCATTTGCAGACAGGTC-3' and reverse, 5'-GCTACACAG CAGCCAGATTCC-3'); GAPDH (forward, 5'-TCAACGGAT TTGGTCGTATTG-3' and reverse, 5'-TGGGTGGAATCATAT TGGAAC-3'); and VEGF-C (forward, 5'-TGTGTGTCCGTC TACAGATGTG-3' and reverse, 5'-TCGGCAGGAAGTGTG ATTGG-3'). Relative expression levels were calculated using the $2^{-\Delta \Delta \mathrm{Cq}}$ method and the specificity of PCR products was verified through $2 \%$ agarose gel electrophoresis. The main steps of western blot analysis were performed according to standard procedures as previously described (24). The kits containing the nuclear and cytoplasmic extraction reagents were purchased from KeyGen Biotech, Co., Ltd. (Nanjing, China).

Cell proliferation assay. Cell proliferation was assessed by the Cell Counting Kit-8 (CCK-8) reagent according to the manufacturer's protocol (Biosharp, Hefei, China). The highly water-soluble tetrazolium salt of CCK-8 kit, WST-8, is reduced by dehydrogenase activities in cells to produce a yellow colored formazan dye, which is soluble in the culture media and directly proportional to the number of living cells. Briefly, the cells were seeded onto 96-well plates at a density of $5 \times 10^{3}$ cells $/ \mathrm{ml}$ and allowed to adhere for $24 \mathrm{~h}$. After 24,48 or $72 \mathrm{~h}$ of incubation, $10 \mu \mathrm{l}$ of CCK-8 solution was added to each well, and the cells were incubated at $37^{\circ} \mathrm{C}$ for $1 \mathrm{~h}$. The optical density (OD) was measured at an absorbance wavelength of $450 \mathrm{~nm}$ in a microplate reader (Bio-Tek, Winooski, VT, USA). All experiments were conducted at least in triplicate. 
Cell migration and invasion assay. For migration analysis, $4 \times 10^{5}$ ECA109 cells/well were plated onto 24 -well plates with complete medium. After scratching with a $10-\mu 1$ pipette tips, followed by pretreatment with let-7a mimics or NC for $24 \mathrm{~h}$, the cells were washed twice with PBS, cultured in serum-free medium, and photographed by a microscope (Olympus, Tokyo, Japan) after 24 and $48 \mathrm{~h}$. Prior to the Transwell migration assay, ECA-109 cells were cultured with or without let-7a mimic for $24 \mathrm{~h}$. Subsequently, the cells were digested, resuspended and seeded at a density of $\sim 2 \times 10^{5}$ of cells/well into the upper chambers, harboring a polycarbonate membrane $(8 \mu \mathrm{m}$ pore size; Corning Incorporated, Corning, NY, USA) containing serum-free medium, while medium containing $10 \%$ FBS was deposited in the lower chambers. Cells that migrated through the membrane were fixed and stained with $0.1 \%$ crystal violet (Solarbio, Beijing, China), and then counted in three randomly selected fields at x200 magnification. The results are expressed as the percentage inhibition rate compared to control.

ELISA and immunofluorescence assays. As previously described, the cell culture supernatants were collected, centrifuged, and immediately stored at $-80^{\circ} \mathrm{C}$ (24). ELISA (Lianke Bio, Hangzhou, China) assay was utilized according to the manufacturer's instructions. For immunofluorescence assays, the cells were fixed and blocked, and successively incubated with primary antibodies against E-cadherin (Cell Signaling Technology, Inc., Beverly, MA, USA) and Vimentin (Boster, Wuhan, China) overnight at $4^{\circ} \mathrm{C}$, and subsequently incubated with $\mathrm{Cy} 3$ or FITC-conjugated secondary antibodies (BD, Biosciences, CA, USA) at room temperature for $1 \mathrm{~h}$. The cells grown on coverslips were counterstained with DAPI (Sigma-Aldrich, St. Louis, MO, USA) and randomly imaged using a fluorescence microscope (Olympus).

Statistical analysis. The results obtained from multiple experiments were reported as the means \pm standard deviation. SPSS 16.0 software was used for data handling, analysis, and presentation. Distinctions between groups were analyzed by Student's t-test or one-way analysis of variance (ANOVA) with post hoc Student-Newman-Keuls test. Statistical significance was set as $\mathrm{P}<0.05$.

\section{Results}

The expression of let-7a is repressed in tissues of ESCC patients. We first investigated the expression of let-7 family members, including let-7a, let-7b and let-7c, in 70 pair of surgically resected human ESCC and para-carcinoma normal tissues by RT-qPCR analysis. The expression of let-7a, let-7b and let-7c were apparently lower in tumors from ESCC patients, among which a relative stable result showed that let-7a may be more representative and focused $(\mathrm{P}=0.0089$; $\mathrm{P}=0.0431$; $\mathrm{P}=0.0397$ ) (Fig. 1A). To verify the specificity of RT-qPCR products, we then categorized the specimens into two groups based on recurrence and detected these products by agarose gel electrophoresis. As shown in Fig. 1B, the let-7a target specificity band emerged at the $100 \mathrm{bp}$ position and showed more expression of let-7a among para-carcinoma normal tissues no matter whether recurrence from ESCC patients. We next investigated the association between let-7a and recurrence. The

Table I. Correlation between let-7a and clinic-pathological features of ESCC patients.

\begin{tabular}{lcc}
\hline $\begin{array}{l}\text { Clinicopathological } \\
\text { features }\end{array}$ & $\begin{array}{c}\text { let-7a } \\
\text { (fold change) }\end{array}$ & P-value
\end{tabular}

\section{Sex}

Male

Female

Age, years

$\geq 50$

$<50$

Diameter of tumor, $\mathrm{cm}$

$>4$
$\leq 4$

Differentiation

High

Middle

Low

TNM stage ${ }^{a}$

$\begin{array}{ll}\text { I/II } & 47 \\ \text { III/IV } & 23\end{array}$

Location

Upper

Middle

Lower

Recurrence $^{\mathrm{a}}$

Yes

No

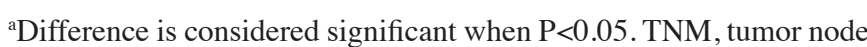
metastasis; ESCC, esophageal squamous cell carcinoma.

expression of let-7a was significantly decreased in the tumor tissues with or without recurrence compared to that of matched normal tissues $(\mathrm{P}<0.01 ; \mathrm{P}=0.0033 ; \mathrm{P}=0.0017)$. Furthermore, the expression of let-7a was significantly lower in the tumor tissues with recurrence than in those without recurrence $(\mathrm{P}<0.05 ; \mathrm{P}=0.0120$; Fig. 1C). The statistical results showed that the expression of let-7a in ESCC tissues was decreased compared to that in the matched normal tissues of 70 ESCC samples and was significantly correlated with advanced stage and tumor recurrence (Table I), indicating that the low expression of let-7a in most human ESCC cases might be involved in the invasion, metastasis and poor prognosis of ESCC.

Repression of let-7a increases the motility of ESCCs in vitro. To determine the relationship of let-7a expression with the malignancy of ESCC, we next examined the expression of let-7a among Het-1a cells and ESCC cell lines by RT-qPCR (Fig. 2A). Compared with Het-1a, the expression of let-7a was significantly lower for ESCC cells $(\mathrm{P}<0.01 ; \mathrm{P}=0.0314 ; \mathrm{P}=0.0130 ; \mathrm{P}=0.0219)$. Nevertheless, there were no significant differences among TE-1, ECA109 and KYSE-150 cells $(\mathrm{P}>0.05 ; \mathrm{P}=0.1192)$. Previous studies have indicated that let- 7 represses the proliferation 
A
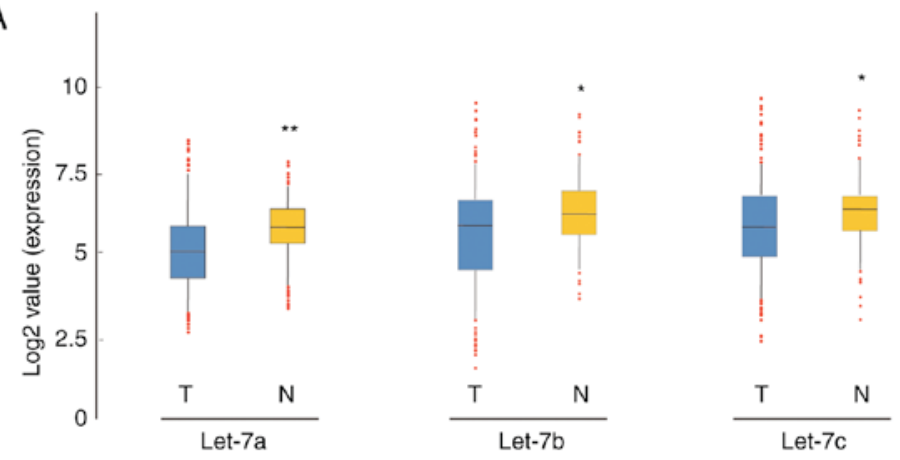

B

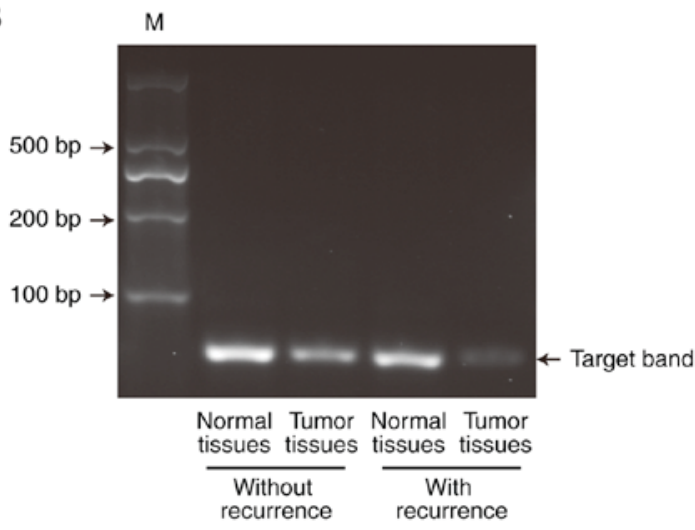

C

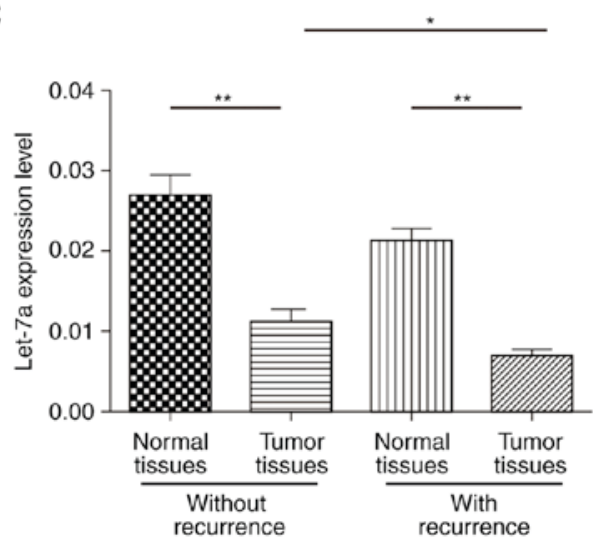

Figure 1. The expression of let-7a in tumors and para-carcinoma tissues of ESCC. (A) The expression level of let-7a, let-7b and let-7c obtained from tumor (T) and normal tissues (N) from 70 ESCC patients by RT-qPCR analysis. (B) The specificity of let-7a in tumors and normal tissues of ESCC (random samples including recurrence or not) was verified through agarose gel electrophoresis. (C) The let-7a expression of tumor and normal tissues in pair (70 cases of ESCC patients) was analyzed by RT-qPCR, which normalized to U6 expression. ${ }^{* *} \mathrm{P}<0.01,{ }^{*} \mathrm{P}<0.05$.

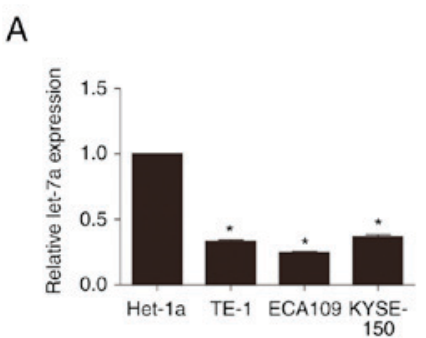

$\mathrm{D}$

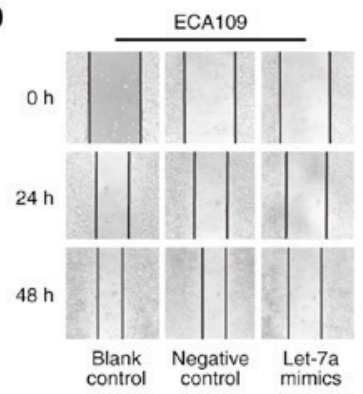

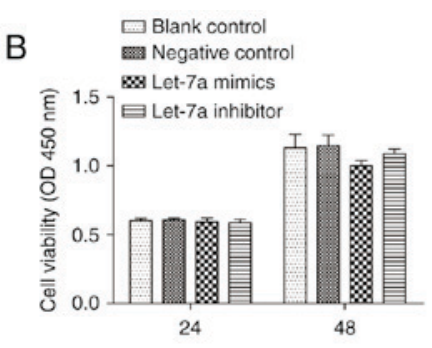

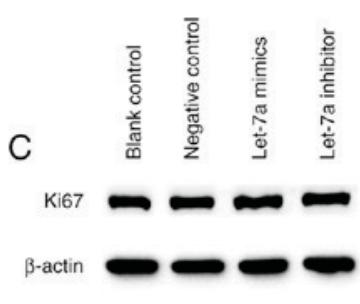

E

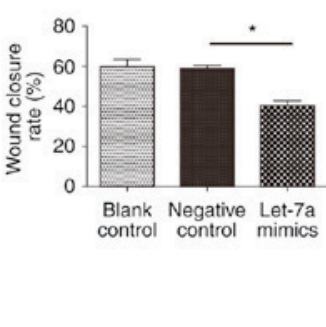

ECA109

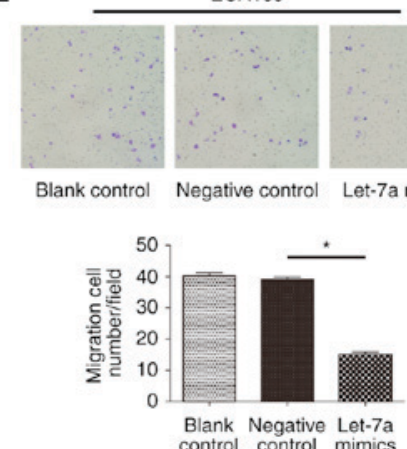

Figure 2. Effect of let-7a expression on the motility of ESCCs. (A) Expression of let-7a in ESCC cell lines compared with Het-1a cells was detected by RT-qPCR. (B) CCK-8 assay were performed to ECA109 cells after let-7a-mimics/inhibitor or NC transfection at 24 and 48 h. (C) The levels of Ki67 protein were measured by western blotting after treatments for $48 \mathrm{~h}$. (D) Representative graphs of wounds after let-7a mimics or NC treatment compared to the control are shown, and the migration rate was quantified in the chart on the right. (E) Representative images of crystal violet-stained migrated cells were captured after $24 \mathrm{~h}$ (magnification, x200). The bottom chart showed the invasion number of ECA109 cells after transfection with let-7a mimics or NC, or no-transfected treatment. NC, negative control. $\mathrm{P}<0.05$.

of ECA109 cells (29). To examine whether let-7a-mediated inhibition affected cell motility, we determined proliferation by CCK-8 assay in ESCC cells transfected with let-7a mimics or inhibitors. Conversely, there was no significant difference 
A

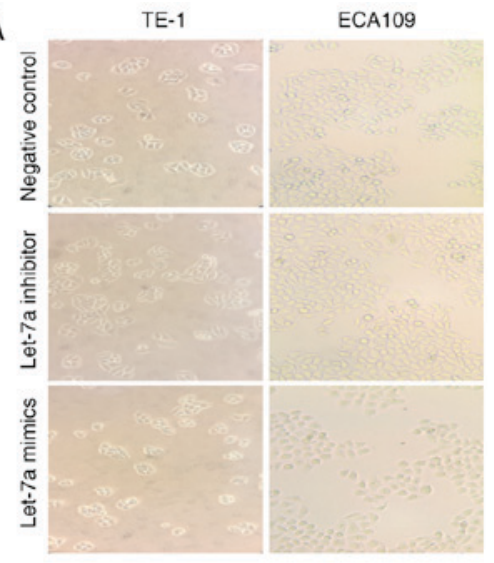

C

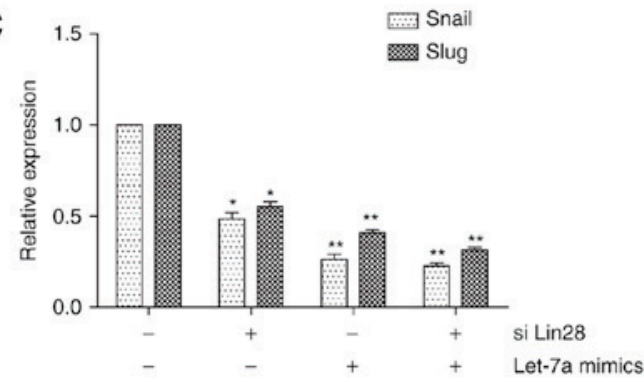

B
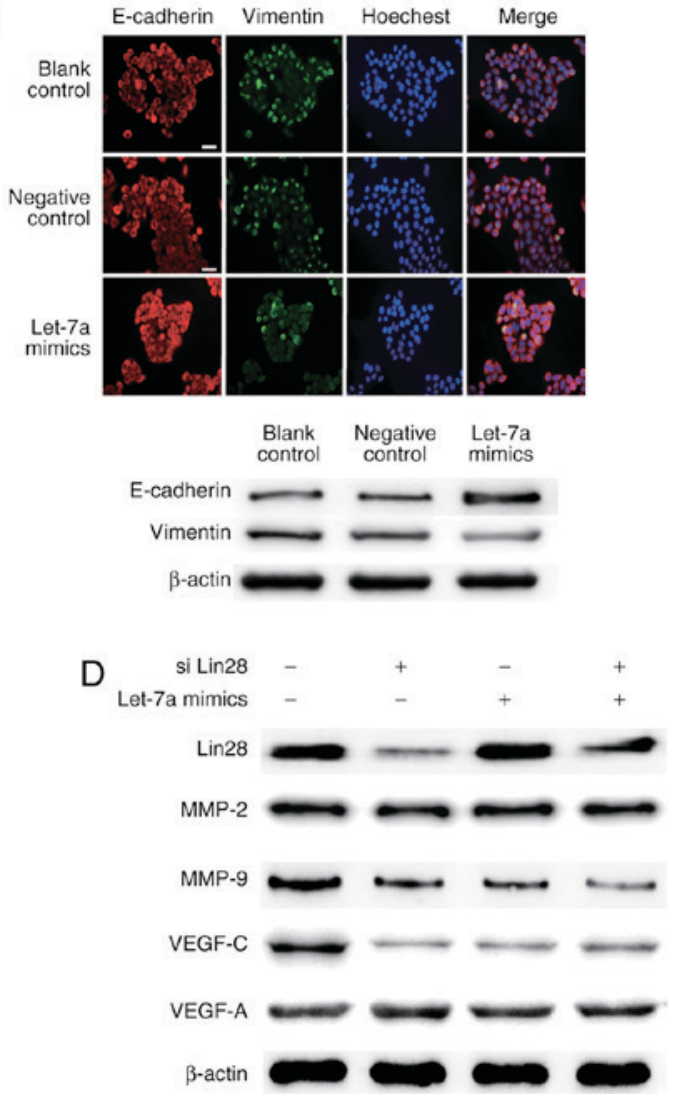

E

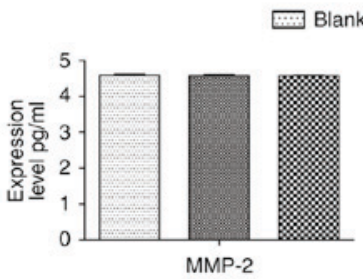

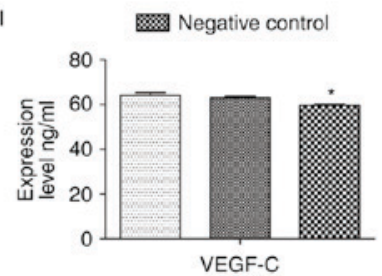

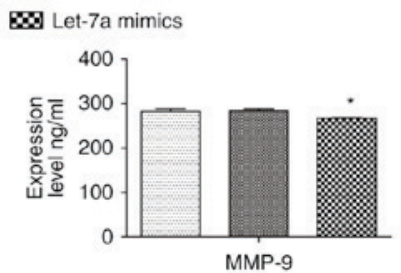

Figure 3. Regulation of Lin28/let-7a on EMT and spreading in ESCCs. (A) Representative images of cell morphology performed in ESCC cells transfected with negative control, let-7a inhibitors or let-7a mimic (magnification, x100). (B) ECA109 cells were transfected with let-7a mimics or NC for $24 \mathrm{~h}$ and then stained for E-cadherin (red), Vimentin (green), and cell nuclei (blue) (scale bar, $50 \mu \mathrm{m}$ ). The changes of E-cadherin and Vimentin were measured by western blotting. (C) The relative expression of slug and snail was detected by RT-qPCR after ECA109 cells were transfected with siLin28 and (or) let-7a mimics. (D) The expression changes of Lin28, VEGF-C, MMP2, MMP9, and VEGF-A after treatment with siLin28 and (or) let-7a mimics for 48 h. (E) ELISA assay measured the secreted VEGF-C, MMP2, and MMP9 after transfection or not for $48 \mathrm{~h}$ in ECA109 cells. siLin28, siRNA-Lin28 treatment. ${ }^{* *} \mathrm{P}<0.01$, ${ }^{*} \mathrm{P}<0.05$.

among treatment groups of ECA109 cells at 24 and $48 \mathrm{~h}$ ( $\mathrm{P}>0.05$; $\mathrm{P}=0.0120$ ) (Fig. 2B), including TE-1 and KYSE-150 cells, even at $72 \mathrm{~h}$ (data not shown). Furthermore, differences in let-7a expression in ECA109 cells showed no significant change in Ki67 (a nuclear protein associated with proliferation) production, compared to control (Fig. 2C). These data indicated that let-7a has no effect on the proliferation of ESCCs. To address the effect of let-7a on cell mobility in vitro, we performed the wound healing and Transwell migration assays. The results showed that let-7a mimics could markedly decrease the cell migration rate at the edge of the scratch (Fig. 2D; $\mathrm{P}=0.0232$ ). Moreover, the migration assay also showed that the invasiveness of cells was significantly restrained after treatment with let-7a mimics (Fig. 2E; $\mathrm{P}=0.0103$ ). However, inhibitors of let-7a had no evident effects (data not shown), potentially due to the extremely low inherent expression of the molecules. These results indicated that let-7a regulated the motility of ESCC cells in vitro, independent of cell proliferation.
Lin28/let-7a regulates the progress of EMT and spreading in ESCCs. EMT is a well-known inducer of tumor metastasis, and we thus speculated that let-7a also acts as a repressor of this progress. Although there were no significant changes in cell morphologies after up or downregulated let-7a expression (Fig. 3A), the results of immunofluorescence and immunoblotting assays showed that ECA109 cells treated with let-7a mimics had an inhibited Vimentin expression and increased E-cadherin expression, indicating that let-7a was involved in the stabilization of EMT (Fig. 3B). We also examined the effect of Lin-28, as an inhibitor of let-7a, on EMT in the presence or not of let-7a mimics. The overexpression of let-7a inhibited the transcription of snail and slug, and the interference of Lin28 showed the same but relatively weaker effect; thus, the combined treatment of both let-7a overexpression and Lin28 interference caused cumulative effects (Fig. 3C; the downregulation of snail was 53.3 \pm 0.74 ; $72.2 \pm 0.81 ; 72.4 \pm 0.69 \%$ respectively; the downregulation of slug was $49.9 \pm 0.66 ; 54.2 \pm 0.88 ; 69.9 \pm 0.77 \%$, respectively). 
A
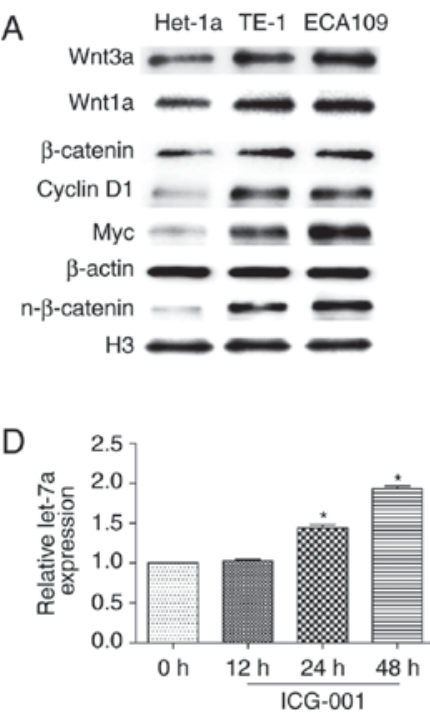

B

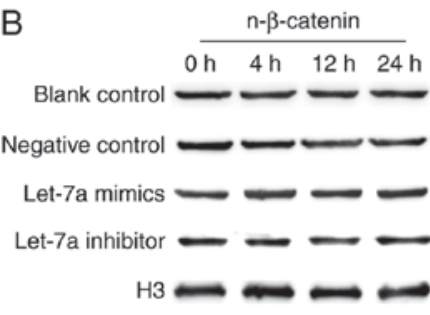

$\mathrm{E}$

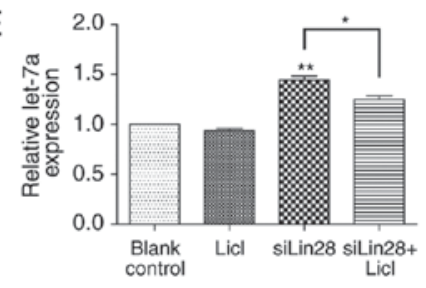

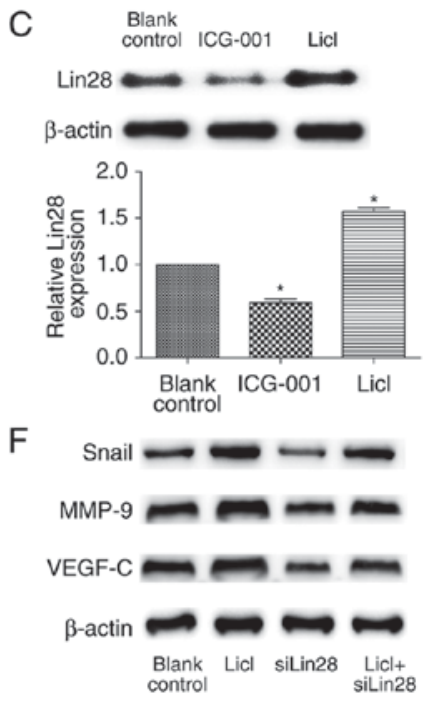

Figure 4. Hyper-activation of Wnt/ $\beta$-catenin suppresses let-7a by Lin28. (A) The activation of Wnt pathway between ESCC cells and Het-1a cells. The western blot results were normalized to $\beta$-actin or Histone 3 as a control. (B) The expression and transposition of $\beta$-catenin were detected by western blotting among let-7a mimics, inhibitors or NC groups at 4, 12 and $48 \mathrm{~h}$. (C) The mRNA and protein levels of Lin28 were measured after ICG-001 (10 $\mu \mathrm{M})$ and LiCl (20 mM) treatment in ECA109 cells at 24 and 48 h. (D) The expression of let-7a was evaluated after ICG-001 (10 $\mu \mathrm{M})$ treatment at 12, 24 and 48 h. (E) ECA109 cells were treated by $\mathrm{LiCl}(20 \mathrm{mM})$ and/or siLin 28 for $24 \mathrm{~h}$, and then the expression of let-7a was evaluated by RT-qPCR. (F) Western blotting showing the changes of snail, VEGF-C and MMP9 expression in ECA109 cells treated with $\mathrm{LiCl}(20 \mathrm{mM})$ and/or siLin28 treatment for 48 h. H3, Histone 3; siLin28, siRNA-Lin28 treatment. ${ }^{*} \mathrm{P}<0.05,{ }^{* *} \mathrm{P}<0.01$.

However, the enhanced let-7a expression did not affect the expression of Lin28. In addition, we further investigated the levels of VEGF-C, VEGF-A, MMP2 and MMP9, which are widely considered as key indicators of metastasis. Similarly, the expression of VEGF-C and MMP9, but not VEGF-A and MMP2, varied inversely with let-7a (Fig. 3D). ELISA results also showed distinct decreases in the levels of VEGF-C $(\mathrm{P}=0.0303)$ and MMP9 $(\mathrm{P}=0.0402)$ expression in the culture supernatant of ECA109 cells, except MMP2 ( $\mathrm{P}=0.6734)$ (Fig. 3E). These findings suggested that let-7a regulates not only the transformation, but also the stromatolysis and vessels regeneration with $\operatorname{Lin} 28$ in ESCCs.

The hyper-activation of Wnt/ $\beta$-catenin suppresses let-7a by Lin28. The Wnt pathway is a key signaling pathway of malignancy; therefore, we assessed the inherent level of $\beta$-catenin among cells. The nuclear-localization expression of $\beta$-catenin was higher in ECA109 and TE-1 cells compared to that in Het-1a cells (Fig. 4A), although there was no statistical significance between both ESCCs (data not shown), and well-known downstream proteins, such as cyclin D1 and Myc, they were synergistically overactive. A recent study demonstrated that let-7a can inhibit EMT through the Wnt pathway in hepatocellular carcinoma stem cells (19); thus, we hypothesized that let-7a may exert the same role in ESCC. Western blot analysis showed that nuclear $\beta$-catenin showed no significant change in ECA109 cells after treatment with let-7a mimics or inhibitors at different times (Fig. 4B). Since Lin28 is a direct downstream gene of the Wnt pathway $(25,26)$, we next examined the effect of Wnt activation on Lin28 expression in ESCCs. As shown in Fig. $4 \mathrm{C}, \mathrm{LiCl}(20 \mathrm{mM})$ upregulated Lin 28 biosynthesis, while ICG-001 potently prevented the accumulation of Lin 28 at $10 \mu \mathrm{M}$. In contrast, the expression of let-7a was considerably higher after the addition of ICG-001 at 24 and 48 h $(\mathrm{P}<0.05$;
$\mathrm{P}=0.0301$, with the upregulation of $1.42 \pm 0.19$ fold; $\mathrm{P}=0.0163$, with the upregulation of $1.91 \pm 0.20$ fold) (Fig. 4D). Next, we detected the expression of let-7a after treatment with $\mathrm{LiCl}$ and/or siRNA-Lin28, and compared with $\mathrm{LiCl}$ treatment, there was a significant decrease of let-7a under the interference of $\operatorname{Lin} 28$ $(\mathrm{P}=0.0412)$ (Fig. 4E). Similarly, the downregulation of Snail, VEGF-C, and MMP9 after Lin28 knockdown was reverted by $\mathrm{LiCl}$ treatment (Fig. 4F). In conclusion, the activation of the Wnt pathway suppresses let-7a by Lin28 in ESCCs.

\section{Discussion}

Let-7 has been recognized as one of the most prominent miRNAs implicated in human malignancy (10-20). The expression of let-7a, let-7b, and let-7c or other members was previously identified as significantly reduced in tumors, and the upregulation of these miRNAs inhibited the process of proliferation, EMT or metastasis. (18-20). In our study, we found low expression of let-7a, let-7b and let-7c in ESCC tumors and that let-7a was significantly inversely correlated with advanced stage, recurrence and poor prognosis. Therefore, the identification of let-7a would definitely be helpful for the clinical decision and management of ESCC. In vitro, the upregulation of let-7a by mimics could markedly decrease the migration and invasiveness rate, but it had no effect on proliferation in ESCCs. According to the initial role of EMT for metastasis, our experiment further revealed the accumulation of E-cadherin and weaker accumulation of Vimentin, with distinct decreases of snail and slug when let-7a was highly expressed. Moreover, the regulation of VEGF-C and MMP9 were also dependent on let-7a levels. This evidence indicated that the loss of let-7a has potential to detect early signals of carcinogenic exposures for ESCC.

The Wnt/ $\beta$-catenin pathway is involved in the development and homeostasis of many normal organs or tissues, thus 
its dysfunction often leads to terrible consequences, such as tumors $(19,21-23,25-27)$. Our previous study indicated that the hyper-activation of Wnt/ $\beta$-catenin pathway stimulated by IL-23 could promote EMT in ESCCs (24). Although recent evidence has shown that let-7a inhibited the Wnt pathway in HCC stem cells (19), the same character has not been confirmed in ESCCs. Furthermore, we found that the inhibition of the Wnt pathway decreased the level of Lin 28 and let-7a restoration. In addition, as the negative regulator of let-7a, Lin28 was selected to observe the impact of Wnt signaling on promoting stromatolysis and vessels regeneration. As expected, after stimulation of the Wnt pathway by $\mathrm{LiCl}$, malignancy was disrupted by siRNA-Lin28 or let-7a-mimic pretreatment. In this study, since the abnormal activation of Wnt/ $\beta$-catenin signaling in ESCC is inevitable, the status of let-7a mediated by Lin 28 may represent dynamic progression.

In conclusion, our results revealed that compared to paracarcinoma tissues, the suppression of let-7a in tumors, is closely associated with the invasion, metastasis and poor prognosis of ESCC. In addition, Wnt/ $\beta$-catenin/Lin 28 signaling induced EMT, and metastasis would occur through the elimination of let-7a. As a screening test for miRNA expression, such as serum, could be easily obtained, these findings provide a novel factor for the prognosis of ESCC in the future.

\section{Acknowledgements}

The present study was supported by the National Natural Science Foundation of China (nos. 81572956 and 81370889), and the Wu Jieping Medical Foundation (320.6755.15022).

\section{References}

1. Ferlay J, Soerjomataram I, Dikshit R, Eser S, Mathers C, Rebelo M, Parkin DM, Forman D and Bray F: Cancer incidence and mortality worldwide: Sources, methods and major patterns in GLOBOCAN 2012. Int J Cancer 136: E359-E386, 2015.

2. Castillo A, Aguayo F, Koriyama C, Torres M, Carrascal E, Corvalan A, Roblero JP, Naquira C, Palma M, Backhouse C, et al: Human papillomavirus in esophageal squamous cell carcinoma in Colombia and Chile. World J Gastroenterol 12: 6188-6192, 2006.

3. Chang D and Church J: Evaluating the health-related quality of life of esophageal cancer patients. Pract Radiat Oncol 4: 181-186, 2014.

4. Ekman S, Dreilich M, Lennartsson J, Wallner B, Brattström D, Sundbom M and Bergqvist M: Esophageal cancer: Current and emerging therapy modalities. Expert Rev Anticancer Ther 8: 14331448,2008

5. Dreikhausen L, Blank S, Sisic L, Heger U, Weichert W, Jäger D, Bruckner T, Giese N, Grenacher L, Falk C, et al: Association of angiogenic factors with prognosis in esophageal cancer. BMC Cancer 15: 121, 2015.

6. Jung HY, Fattet L and Yang J: Molecular pathways: Linking tumor microenvironment to epithelial-mesenchymal transition in metastasis. Clin Cancer Res 21: 962-968, 2015.

7. Brabletz S and Brabletz T: The ZEB/miR-200 feedback loop-a motor of cellular plasticity in development and cancer? EMBO Rep 11: 670-677, 2010.

8. Chhabra R and Saini N: MicroRNAs in cancer stem cells: Current status and future directions. Tumour Biol 35: 8395-8405, 2014

9. Rajasekaran S, Rajaguru P and Sudhakar Gandhi PS: MicroRNAs as potential targets for progressive pulmonary fibrosis. Front Pharmacol 6: 254, 2015.

10. Lin S and Gregory RI: MicroRNA biogenesis pathways in cancer. Nat Rev Cancer 15: 321-333, 2015.

11. Yang G, Zhang W, Yu C, Ren J and An Z: MicroRNA let-7: Regulation, single nucleotide polymorphism, and therapy in lung cancer. J Cancer Res Ther 11 (Suppl 1): C1-C6, 2015.
12. Alajez NM, Shi W, Wong D, Lenarduzzi M, Waldron J, Weinreb I and Liu FF: Lin 28 b promotes head and neck cancer progression via modulation of the insulin-like growth factor survival pathway. Oncotarget 3: 1641-1652, 2012.

13. Ohshima K, Inoue K, Fujiwara A, Hatakeyama K, Kanto K, Watanabe Y, Muramatsu K, Fukuda Y, Ogura S, Yamaguchi K and Mochizuki T: Let-7 microRNA family is selectively secreted into the extracellular environment via exosomes in a metastatic gastric cancer cell line. PLoS One 5: e13247, 2010.

14. Boyerinas B, Park SM, Murmann AE, Gwin K, Montag AG, Zillhardt M, Hua YJ, Lengyel E and Peter ME: Let-7 modulates acquired resistance of ovarian cancer to Taxanes via IMP-1-mediated stabilization of multidrug resistance 1. Int J Cancer 130: 1787-1797, 2012.

15. Sugimura K, Miyata H, Tanaka K, Hamano R, Takahashi T, Kurokawa Y, Yamasaki M, Nakajima K, Takiguchi S, Mori M and Doki Y: Let-7 expression is a significant determinant of response to chemotherapy through the regulation of IL-6/STAT3 pathway in esophageal squamous cell carcinoma. Clin Cancer Res 18: 5144$5153,2012$.

16. Hamano R, Miyata H, Yamasaki M, Sugimura K, Tanaka K, Kurokawa Y, Nakajima K, Takiguchi S, Fujiwara Y, Mori M and Doki Y: High expression of Lin28 is associated with tumour aggressiveness and poor prognosis of patients in oesophagus cancer. $\mathrm{Br} \mathrm{J}$ Cancer 106: 1415-1423, 2012.

17. Liu Y, Li H, Feng J, Cui X, Huang W, Li Y, Su F, Liu Q, Zhu J, Lv X, et al: Lin 28 induces epithelial-to-mesenchymal transition and stemness via downregulation of let-7a in breast cancer cells. PLoS One 8: e83083, 2013.

18. Wu A, Wu K, Li J, Mo Y, Lin Y, Wang Y, Shen X, Li S, Li L and Yang Z: Let-7a inhibits migration, invasion and epithelialmesenchymal transition by targeting HMGA2 in nasopharyngeal carcinoma. J Transl Med 13: 105, 2015.

19. Jin B, Wang W, Meng XX, Du G, Li J, Zhang SZ, Zhou BH and Fu ZH: Let-7 inhibits self-renewal of hepatocellular cancer stemlike cells through regulating the epithelial-mesenchymal transition and the Wnt signaling pathway. BMC Cancer 16: 863, 2016.

20. Li B, Chen P, Chang Y, Qi J, Fu H and Guo H: Let-7a inhibits tumor cell growth and metastasis by directly targeting RTKN in human colon cancer. Biochem Biophys Res Commun 478: 739-745, 2016.

21. Wang Y, Shi J, Chai K, Ying X and Zhou BP: The role of snail in EMT and tumorigenesis. Curr Cancer Drug Targets 13: 963-972, 2013.

22. Nusse R: Wnt signaling and stem cell control. Cell Res 18: 523-527, 2008.

23. Sun X, He Y, Huang C, Ma TT and Li J: Distinctive microRNA signature associated of neoplasms with the Wnt/ $\beta$-catenin signaling pathway. Cell Signal 25: 2805-2811, 2013.

24. Chen D, Li W, Liu S, Su Y, Han G, Xu C, Liu H, Zheng T, Zhou Y and Mao C: Interleukin-23 promotes the epithelial-mesenchymal transition of oesophageal carcinoma cells via the $\mathrm{Wnt} / \beta$-catenin pathway. Sci Rep 5: 8604, 2015.

25. Tu HC, Schwitalla S, Qian Z, LaPier GS, Yermalovich A, Ku YC, Chen SC, Viswanathan SR, Zhu H, Nishihara R, et al: LIN28 cooperates with WNT signaling to drive invasive intestinal and colorectal adenocarcinoma in mice and humans. Genes Dev 29: 1074-1086, 2015.

26. Cai WY, Wei TZ, Luo QC, Wu QW, Liu QF, Yang M, Ye GD, Wu JF, Chen YY, Sun GB, et al: The Wnt $\beta \beta$-catenin pathway represses let-7 microRNA expression through transactivation of Lin28 to augment breast cancer stem cell expansion. J Cell Sci 126: 2877-2889, 2013.

27. Li J, Ying J, Fan Y, Wu L, Ying Y, Chan AT, Srivastava G and Tao Q: WNT5A antagonizes WNT/ $\beta$-catenin signaling and is frequently silenced by promoter $\mathrm{CpG}$ methylation in esophageal squamous cell carcinoma. Cancer Biol Ther 10: 617-624, 2010.

28. Gao J, Li N, Dong Y, Li S, Xu L, Li X, Li Y, Li Z, Ng SS, Sung JJ, et al: miR-34a-5p suppresses colorectal cancer metastasis and predicts recurrence in patients with stage II/III colorectal cancer. Oncogene 34: 4142-4152, 2015.

29. Liu Q, Lv GD, Qin X, Gen YH, Zheng ST, Liu T and Lu XM: Role of microRNA let-7 and effect to HMGA2 in esophageal squamous cell carcinoma. Mol Biol Rep 39: 1239-1246, 2012.

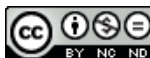

This work is licensed under a Creative Commons Attribution-NonCommercial-NoDerivatives 4.0 International (CC BY-NC-ND 4.0) License. 